Supplement of Biogeosciences Discuss., 12, 19711-19750, 2015

http://www.biogeosciences-discuss.net/12/19711/2015/

doi:10.5194/bgd-12-19711-2015-supplement

(C) Author(s) 2015. CC Attribution 3.0 License.

(c) (1)

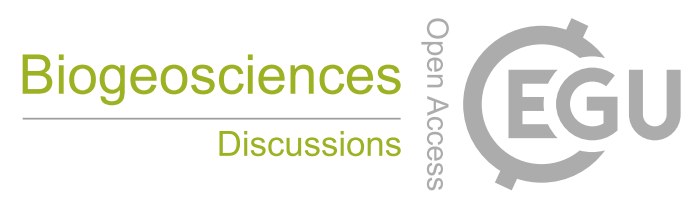

Supplement of

\title{
Closing a gap in tropical forest biomass estimation: accounting for crown mass variation in pantropical allometries
}

\section{P. Ploton et al.}

Correspondence to: P. Ploton (pierre.ploton@ird.fr)

The copyright of individual parts of the supplement might differ from the CC-BY 3.0 licence. 


\section{Supplement}

(

\section{S1 Field data protocols}

\section{S1.1 Unpublished dataset: site characteristics}

Field work was conducted close to the city of Mindourou-2 $\left(4^{\circ} 7^{\prime}, 14^{\circ} 32^{\prime} \mathrm{E}\right)$ in the logging concessions of Alpicam-Grumcam Company (67 trees) and approximately $150 \mathrm{~km}$ southwest of this location, in community forests (10 trees) surrounding the city of Lomie $\left(3^{\circ} 9^{\prime} \mathrm{N}\right.$, $\left.13^{\circ} 37^{\prime} \mathrm{E}\right)$. In both locations, the vegetation type can be classified as semi-deciduous Celtis forest (sensu Fayolle et al. 2014). The average annual rainfall of the area is 1500-2000 mm with two marked dry seasons, from mid-November to mid-March (long dry season) and from June to mid-August (small dry season). The average annual temperature is approximately 24 ${ }^{\circ} \mathrm{C}$. The elevation ranges between 600 and $700 \mathrm{~m}$ a.s.l.

\section{S1.2 Biomass data}

\section{S1.2.1 Unpublished dataset}

A first set of 67 trees were felled as part of the routine activities of a logging company. Tree sampling targeted large individuals of 10 abundant species. For a second set of 10 trees, we used a less destructive protocol consisting in volume measurements on standing trees by expert tree climbers.

In both felled and standing trees, the volume of the largest components of tree structure (i.e., buttresses, stumps, trunk and large branches, namely those with a sectional diameter - or $D b$ for branch diameter - greater than $20 \mathrm{~cm}$ ) was estimated following Henry et al. (2009). For the trunk, we measured the proximal and distal diameters of approximately 2-m long conical sections and applied Smalian's formula to compute the volume of each section. A similar procedure was used for large branches, with the exception that conical sections were approximately $1 \mathrm{~m}$ long. Buttress volumes were estimated using the dedicated formula reported by Henry et al. (2009). On felled trees, 5-cm-thick wood slices were collected at the top of stumps and trunks and in large branches. Three parallelepipeds of approximately $5 * 5$ $* 2.5 \mathrm{~cm}$ were then sampled radially from each slice at the sawmill. The wood density $(\rho)$ of each parallelepiped sample was determined from its green volume (waster displacement method) and oven-dried mass (Williamson et Wiemann 2010). Analyses of wood density variations revealed significant species, individual and vertical (i.e., stump, buttresses and 
trunk vs large branches) effects (result not shown). We therefore converted the volume of stumps, buttresses and trunks to dry mass using an individual average of $\rho$ estimates in these components. The volumes of large branches were converted to dry mass using individual averages of $\rho$ estimates in large branches. For standing trees, volume estimates of all components were converted to mass using individual $\rho$ values obtained from a single pruned branch $(10 \leq D b \leq 20 \mathrm{~cm})$.

The dry mass of small branches $(D b \leq 20 \mathrm{~cm})$ was estimated using a different protocol. On each tree, the total fresh mass and the leaf fresh mass of one to three damage-free branches were weighted, and their proximal diameter measured. From the resulting database, we built a mixed-species linear model relating branch diameter to total fresh mass (in logarithmic units). For some species presenting a significant main species effect, a species-specific model was developed (results not shown). These models were used to compute the total fresh mass of small branches $(D b \leq 20)$ that were not directly weighted in the field. We then established linear models relating small branch total fresh mass to leaf fresh mass with a similar procedure. The latter models were used to decompose small branch total fresh mass predictions into leaf and wood fresh masses. Approximately $200 \mathrm{~g}$ of leaves per sample branch were oven-dried to determine a species-specific fresh to dry leaf mass conversion ratio. For each tree, a wood slice was collected from a sampled small branch and $\rho$ was determined as previously described, allowing the conversion of small branch wood fresh mass to dry mass.

The total $A G B$ of a tree $(T A G B)$ was obtained by summing the dry masses of the stump, buttresses, trunk, large branches, woody parts of small branches and leaves.

In addition to basic dendrometric measurements $(D, H)$ and full crown structure description (branch diameters, lengths and topology), two perpendicular crown diameters were measured using a Laser Ranger-finder device (TruPulse 360R, Laser Technology Inc., Centennial, Colorado) for 39 individuals.

\section{S1.2.2 Other datasets}

We additionally compiled destructive datasets providing information on crown mass for 29 trees from Ghana (Henry et al. 2010), 285 trees from Madagascar (Vieilledent et al. 2011), 51 trees from Peru (Goodman, Phillips \& Baker 2014, 2013), 132 trees from Cameroon (Fayolle et al. 2013), and 99 trees from Gabon (Ngomanda et al. 2014). In the dataset from Ghana, we 


\begin{tabular}{llccc}
\multicolumn{1}{c}{ Source } & Country & Data $_{\text {CM1 }}$ & Data & Data \\
\hline P. Ploton & Cameroon & 77 & 77 & 39 \\
Henry et al. (2010) & Ghana & 29 & 29 & 29 \\
Goodman et al. (2013) & Peru & 51 & 51 & 51 \\
Fayolle et al. (2013) & Cameroon & 132 & & \\
Ngomanda et al. (2014) & Gabon & 99 & 99 & \\
Vieilledent et al. (2012) & Madagascar & 285 & 285 & \\
& & 673 & 541 & 119
\end{tabular}

\section{S1.3 Inventory data}

In all plots, we considered all trees with a diameter at breast height (i.e., $1.3 \mathrm{~m}$ or above buttresses if present) $\geq 10 \mathrm{~cm}$. In the 801 -ha plots, tree height was measured with a Laser Ranger-finder device (TruPulse 360R, Laser Technology Inc., Centennial, Colorado) on approximately 50 trees per plot, homogenously distributed across diameter classes. Following 
1 Feldpausch et al. (2012), a three-parameter Weibull function was fitted at the site level to 2 predict height of the remaining trees: $H=a\left(1-\exp \left(-b D^{c}\right)\right)$. We used a relationship 3 calibrated over two 1-ha plots near Korup to predict tree heights in the 50-ha permanent plot. 4 Trees were identified in the field by expert botanists, and herbarium specimens were collected 5 on each species per site for cross-identification at the herbarium of Universite Libre de 6 Bruxelles (BRLU), except for Korup, where the taxonomy was confirmed at the Missouri 7 Botanical Garden (MO). Of 48,155 measured trees, $88.4 \%$ were identified at the species level, $84.9 \%$ at the genus level, and $0.1 \%$ at the family level, and $6.4 \%$ were left unidentified. We 9 used the Dryad Global Wood Density Database (Chave et al. 2009; Zanne et al. 2009) to 10 attribute to each individual tree a wood density value. For species known only at the genus or family level, the average $\rho$ value at that taxonomic level was used (Chave et al. 2006). 